\title{
Incidence and management of acute otitis media in adults: a primary care-based cohort study
}

Running title: Acute otitis media in adults

Article category: Epidemiology

\section{Authors}

Merijn H. Rijk ${ }^{1}$, Saskia Hullegie ${ }^{1}$, Anne G.M. Schilder ${ }^{1-3}$, Marlous F. Kortekaas ${ }^{1}$, Roger A.M.J. Damoiseaux ${ }^{1}$, Theo J.M. Verheij ${ }^{1}$, Roderick P. Venekamp ${ }^{1}$

\section{Author affiliations}

${ }^{1}$ Julius Center for Health Sciences and Primary Care, University Medical Center Utrecht, Utrecht University, Utrecht, The Netherlands

${ }^{2}$ National Institute of Health Research, University College London Hospitals Biomedical Research Centre, London, United Kingdom

${ }^{3}$ evidENT, Ear Institute, University College London, London, United Kingdom

\section{Corresponding author}

Mr M. H. Rijk, Julius Center for Health Sciences and Primary Care, University Medical Center Utrecht, Utrecht University, Universiteitsweg 100, Utrecht, 3584 CX, the Netherlands. Email address: merijnrijk@gmail.com.

Key messages 
- Data on the burden of acute otitis media (AOM) among adults in primary care are scarce.

- In Dutch primary care, the incidence of AOM in adults is 5/1000 person-years.

- AOM incidence declines with age and is higher in atopic patients.

- Oral antibiotic prescription rates in adults resemble those in children.

\section{Word count}

Abstract: 223

Main text: 1,980 


\section{ABSTRACT}

Background: Although primarily considered a childhood disease, acute otitis media (AOM) also occurs in adults. Data on the burden of this condition in adults are, however, scarce.

Objective: To explore the primary care incidence and current management of AOM in adults.

Methods: All patients aged 15 and older included in the routine health care database of the Julius General Practitioners' Network were followed from 2015 to 2018 (contributing to a total of $1,261,575$ person-years). We extracted data on AOM episodes, AOM-related consultations, co-morbidities and antibiotic and analgesic prescriptions.

Results: Five thousand three hundred and fifty-eight patients experienced one or more AOM episodes (total number of AOM episodes: 6667; mean 1.2 per patient). The overall AOM incidence was 5.3/1,000 person-years and was fairly stable over the study period. Incidence was particularly high in atopic patients (7.3/1000 person-years) and declined with age (from 7.1 in patients 15-39 years of age to $2.7 / 1000$ person-years in those aged 64 years and older). Oral antibiotics, predominantly amoxicillin, were prescribed in $46 \%$, and topical antibiotics in $21 \%$ of all episodes.

Conclusion: Over the past years, the incidence of AOM in adults in primary care has been stable. Oral antibiotic prescription rates resemble those in children with AOM, whereas a remarkably high topical antibiotic prescription rate was observed. Future prognostic 
research should inform on the need and feasibility of prospective studies into the best management strategy in this condition.

\section{Keywords (MeSH)}

Adult, epidemiology, incidence, otitis media, primary health care, treatment 


\section{Introduction}

Although young children are particularly prone to develop acute otitis media (AOM), it can also occur in adults. Factors associated with the relatively high incidence in children are immaturity of the immune system and the Eustachian tube ${ }^{1-3}$, along with other host and environmental factors such as atopic diathesis and exposure to tobacco smoke.4,5 Complications of AOM are rare in high-income countries, but include serious - and potentially lethal - disease entities such as meningitis and brain abscesses. ${ }^{1}$

To date, direct data on the burden of AOM in adults are scarce; a 2012 systematic review therefore provided a modelling-based incidence of $\mathrm{AOM}$ in this population. ${ }^{6} \mathrm{~A}$ recent publication on $\mathrm{AOM}$ in primary and emergency care settings among a predominantly male cohort of USA veterans reported a mean incidence rate of $2.7 / 1,000$ person-years. ${ }^{7}$ Furthermore, evidence on prognosis and management of AOM is lacking, which leaves general practitioners (GPs) with uncertainty how to best treat adult AOM patients in everyday practice. As a result, GPs may base their treatment decisions on the available clinical guidelines of AOM, which solely derive from research in children. ${ }^{8-10}$

To gain more insight in the burden of AOM among adults, we set out to explore the incidence and current management of AOM in adults in Dutch primary care using 2015 to 2018 routine primary health care data. 


\section{Methods}

\section{Design and patient population}

For this retrospective cohort study, we used pseudonymized routine health care data extracted from the Julius General Practitioners' Network (JGPN) database. ${ }^{11}$ This database covers over 70 primary care practices with around 200 GPs and holds data on patient demographics, consultations, disease episodes (using the International Classification of Primary Care (ICPC) ${ }^{12}$ ) and prescriptions (using Anatomical Therapeutic Chemical (ATC) codes $^{13}$ ). We included all patients aged 15 and older enlisted in the participating practices between 2015 and 2018.

\section{Data extraction}

For all eligible patients, we extracted data on year of birth, gender, diagnosis of atopic diathesis (ICPC R96 (asthma) and/or R97 (allergic rhinitis) and/or S87 (constitutional eczema)), type 1 and 2 diabetes mellitus (T90, which has been associated with increased susceptibility for infections ${ }^{14}$ ), and use of systemic corticosteroids (ATC H02) or other immunosuppressive drugs (ATC L04). In addition, we extracted data on AOM-related prescriptions and number of AOM-related consultations. For oral antibiotics, we only included ATC codes of amoxicillin, amoxicillin/clavulanate, co-trimoxazole, and clarithromycin, since these are mentioned in the 'AOM in children' clinical practice guideline issued by the Dutch College of General Practitioners. ${ }^{8}$ We also included azithromycin, since it has been listed as second-line option until the 2014 update of the 'AOM in children' guideline, and its prescription might have continued into our study period.

\section{Outcome measures}


Our main outcome of interest was GP-diagnosed AOM (ICPC H71). A new AOM episode was defined as an AOM-related primary care consultation after a period of at least 28 days without any AOM-related consultation.

Secondary outcome measures included the proportion of AOM episodes in which (oral and topical) antibiotics and analgesics were prescribed, and the number of consultations per AOM episode. Since disease episodes and prescriptions are not directly linked in the JGPN database, an AOM-related prescription was defined as an antibiotic or analgesic prescribed between 7 days before and after an AOM episode.

\section{Statistical analysis}

Overall and year-specific AOM incidence rates with corresponding 95\% confidence intervals (CIs) were calculated by dividing the number of AOM episodes by the total number of person-years for all adults and stratified according to age (15-39, 40-64, and $\geq 65$ years), gender, comorbidity, and use of systemic corticosteroids or other immunosuppressive drugs prior to an AOM episode. Additionally, we stratified incidence rates in atopic patients according to age to assess whether age modified the observations.

Next, we calculated the number of (oral and topical) antibiotics and analgesics per $100 \mathrm{AOM}$ episodes and the mean number of consultations per AOM episode, all with corresponding 95\% CIs. To assess whether management differed across patient groups, we stratified the results according to the specified age groups, gender, comorbidity, and use of immunosuppressive drugs. In an exploratory analysis, we estimated the consultation rates for patients who did and did not receive an immediate (oral or topical) antibiotic prescription. In line with the explorative nature of our study and due to the large number of subjects, we aimed to describe the observed trends in our database rather than performing formal tests to assess any statistically significant differences for the 
various outcome measures. Statistical analyses were performed using SPSS version 25 (SPSS Inc., Chicago, IL) and Rothman's Episheet version 11 June 2008. 


\section{Results}

\section{Study population}

The size of the cohort varied from 324054 adults in 2015 to 351495 in 2018 contributing to a total follow-up time of 1261575 person-years. Overall, 52.1\% of the patients was female, $45.5 \%$ was aged between 15 and 39 years, 37.6\% between 40 and 64 years, and $16.9 \%$ was 65 or older. On average, $21.4 \%$ of the population had an atopic diathesis and $6.0 \%$ had diabetes. Systemic corticosteroids or other immunosuppressants were used during $0.7 \%$ of all person-years.

\section{AOM episodes}

A total of 5358 patients (58.9\% female) experienced one or more AOM episodes (total number of AOM episodes: 6,667; range 1 to 13; median 1 episode per patient; Table 1).

\section{Incidence rates}

The overall AOM incidence rate was 5.3/1000 person-years (95\% CI: 5.2 to 5.4). Yearly incidence rates revealed a more or less stable pattern. (Figure 1).

Incidence of AOM declined with age and was higher in female patients (Table 2). The presence of an atopic diathesis was associated with a higher AOM incidence rate (Table 2); this observation was independent of age (Table S1).

\section{Prescriptions}

Oral antibiotics were prescribed in $46.0 \%$ of AOM episodes ( $95 \% \mathrm{CI}: 44.8$ to 47.2 ); $84.0 \%$ were immediate prescriptions. In $5.1 \%$ of these episodes, a second course of oral antibiotics was prescribed (Table 3). Amoxicillin was most commonly prescribed (83.8\%), followed by amoxicillin/clavulanate (Figure 2). Antibiotic prescription rates 
were stable over time (Figure 1). No substantial differences in oral antibiotic prescription rates across various subgroups were observed (Table 2).

Overall, topical antibiotics were prescribed in $21.2 \%$ of AOM episodes $(95 \% \mathrm{CI}$ : 20.2 to 22.2 ). Topical antibiotic prescription rates increased with age, ranging from $18.7 \%$ (95\% CI: 17.5 to 20.0 ) in patients aged $15-39$ years to $31.7 \%$ (95\% CI: 28.0 to 35.6 ) in patients aged 65 years and older.

Twelve percent of the episodes in which no immediate oral antibiotic prescription strategy was applied resulted in an oral antibiotic prescription during reconsultation versus $9 \%$ of those initially treated with topical antibiotics (Table 3 ).

Analgesics were prescribed in $8.4 \%$ of AOM episodes (95\% CI: 7.8 to 9.1), mainly non-steroidal anti-inflammatory drugs (NSAIDs, 4.4\%). Cyclooxygenase-2 inhibitors and opioids were prescribed in $2.3 \%$ and $1.6 \%$, respectively.

\section{Consultation rates}

The overall consultation rate in our cohort was 1.5 per AOM episode (95\% CI: 1.5 to 1.6). Consultation rates increased with age (Table 2) and were higher in patients receiving immediate oral and topical antibiotics than in those who did not (Table 3). 


\section{Discussion}

\section{Summary}

Our primary care-based cohort study of routine health care data showed that the incidence rate of AOM in adults is around 5/1000 person-years. The incidence of AOM declined with age and was higher in patients with an atopic diathesis. Oral antibiotics, predominantly amoxicillin, were prescribed in half of the AOM episodes, and topical antibiotics in one-fifth of episodes.

\section{Strengths and limitations}

To the best of our knowledge, our study is one of the first to explore the incidence and management of AOM in adults in primary care. Major strengths are the size of the cohort and data completeness. Since characteristics of all disease episodes in Dutch primary care are systematically registered using ICPC- and ATC-codes, and registration in a primary care practice is mandatory for all Dutch citizens, the JGPN database encompasses recent, well-documented electronic primary health care data. Moreover, the database includes data from primary care practices from both urban and rural areas. The prevalence rates of comorbidities in our cohort closely resemble those of national figures ${ }^{15}$, which further underlines the generalizability of our data.

Some limitations deserve further attention. Most importantly, the use of routine health care data may introduce misclassification of AOM diagnosis and comorbidities. This is particularly relevant to cases for which topical antibiotics were prescribed. These episodes might reflect adults with $\mathrm{AOM}$ and ear discharge due to a spontaneous rupture of the ear drum, which is generally considered a more severe expression of AOM. ${ }^{16}$ There is however no evidence to support the use of topical antibiotics in AOM and ear discharge. ${ }^{17}$ Other possible explanations might be misclassification of ICPC coding or 
disease. When the ear canal is filled with ear discharge it is difficult to distinguish AOM from acute otitis externa, in particular in adults where acute otitis externa is more common. ${ }^{8,18}$

Furthermore, we were not able to extract data on AOM complications requiring specialist referrals since a direct link between ICPC-code and referrals were missing in our database. In addition, exposure to tobacco smoke is a well-known risk factor for AOM in children ${ }^{4,5}$. We were, however, not able to extract valid data on patient's smoking history, due to a substantial number of missing data.

In contrast to the World Allergy Organization's definition of atopy ${ }^{19}$, we did not include proven immunoglobulin E (IgE) sensitization (through elevated IgE levels or a positive skin prick test) in our definition of atopic diathesis, since these tests are not routinely performed in primary care. This may, however, have led to an overestimation of atopic diathesis in our study population.

Inherent to its observational design, our study may suffer from confounding. This is particularly relevant for the observed higher incidence rate in patients with atopic diathesis. Although this observation was consistent across different age groups, we were unable to correct for other (unknown) confounding factors that might have influenced this observation. Confounding by indication particularly applies to our analysis in which we determined the consultation rates of patients who did and did not receive an immediate antibiotic prescription. The observation, i.e. higher consultation rates in those receiving immediate antibiotics, should therefore be interpreted with caution.

\section{Comparison with existing literature}

The AOM incidences observed in our study are in line with those modelled by Monasta et al. ${ }^{6}$ for Western European adults, although they reported a slight increase in incidence in 
those aged 65 years and older. Incidences are also similar to the Dutch 2000-2002 rates which ranged from 8.2/1000 person-years at ages $15-24$ years to 1.7 at ages over $74 .{ }^{15}$

Approximately half of AOM episodes were treated with oral antibiotics, predominantly amoxicillin. These rates are similar to those in Dutch children, with antibiotic prescribing rates ranging from $55 \%$ to $59 \% \cdot{ }^{20,21}$ The antibiotic prescription rate among US veterans was higher, i.e. $75 \%{ }^{7}$, which may be attributed to the difference in study setting and in antibiotic prescribing habits between the USA and the Netherlands.

The very low analgesic prescription rates observed in our study are in agreement with those observed in childhood AOM. ${ }^{22}$ The actual use of analgesics in AOM is probably much higher since paracetamol and most NSAIDs are available over-the-counter.

\section{Implications for practice and further research}

In conclusion, we found that AOM incidence among adults has been stable over the past years at around 5/1000 person-years and that approximately half and one-fifth of the episodes was treated with oral and topical antibiotics, respectively. The observed prescribing of azithromycin and topical antibiotics, to the degree these were actually prescribed for AOM, implies a need for GP-targeted interventions to promote the appropriate use of antibiotics for AOM.

This explorative study serves as a first step in unravelling the burden of AOM among adults in primary care. To determine the need and feasibility of prospective studies into the best management strategy for AOM in adults, further research should focus on factors associated with the prognosis of AOM in terms of symptom duration, complications and need for specialist referrals and/or hospitalization in this patient group. 
Ethical approval By the Medical Ethics Review Committee of the University Medical Centre Utrecht (WAG/mb/19/041366)

Funding No financial support was obtained

Conflicts of interest None declared

Data availability The data underlying this article were provided by the Julius General Practitioners' Network (JGPN) database by permission. Data will be shared on request to the corresponding author with permission of the JGPN.

Acknowledgements The authors thank Marloes M. van Beurden and Nicole Boekema for their contribution by extracting all required data from the JGPN database. 


\section{References}

1. Schilder AGM, Chonmaitree T, Cripps AW, et al. PRIMER: Otitis Media. Nat Rev Dis Prim. 2016;2:1-19.

2. Massa HM, Lim DJ, Kurono Y, Cripps AW. Chapter 101 - Middle Ear and Eustachian Tube Mucosal Immunology. In: Mestecky J, Strober W, Russell MW, Kelsall BL, Cheroutre H, Lambrecht B, eds. Boston: Academic Press; 2015:1923-1942.

3. Chonmaitree T, Revai K, Grady JJ, et al. Viral Upper Respiratory Tract Infection and Otitis Media Complication in Young Children. Clin Infect Dis. 2008;46(6):815-823.

4. Zhang Y, Xu M, Zhang J, Zeng L, Wang Y, Zheng QY. Risk factors for chronic and recurrent otitis media-A meta-analysis. PLoS One. 2014;9(1).

5. Uhari M, Mäntysaari K, Niemelä M. A meta-analytic review of the risk factors for acute otitis media. Clin Infect Dis. 1996;22(6):1079-1083.

6. Monasta L, Ronfani L, Marchetti F, et al. Burden of disease caused by otitis media: Systematic review and global estimates. PLoS One. 2012;7(4).

7. Pontefract B, Nevers M, Fleming-Dutra KE, Hersh A, Samore M, Madaras-Kelly K. Diagnosis and Antibiotic Management of Otitis Media and Otitis Externa in United States Veterans. Open Forum Infect Dis. 2019;(1): 1-7.

8. Damoiseaux, RAMJ; Venekamp, RP; Eekhof, JAH; Bennebroek Gravenhorst, FM; Schoch, AG; Burgers, JS; Bouma, M; Wittenberg J. NHG-Standaard Otitis media acuta bij kinderen. Huisarts Wet. 2014;57(12):648.

9. National Institute for Health and Care Excellence. Otitis media (acute): antimicrobial prescribing (NICE Guideline 91). https://www.nice.org.uk/guidance/ng91. Published 2018. Accessed November 26, 2019.

10. Lieberthal AS, Carroll AE, Chonmaitree T, et al. The diagnosis and management of 
acute otitis media. Pediatrics. 2013;131(3): e964 - 99.

11. Smeets H, Kortekaas M, Rutten F, et al. Routine primary care data for scientific research, quality of care programs and educational purposes: the Julius General Practitioners' Network (JGPN). BMC Heal Serv Res. 2018;18(1):735.

12. World Organization of National Colleges, Academies, and Academic Associations of General Practitioners/Family Physicians. ICPC-2: International Classification of Primary Care. Oxford: Oxford University Press, 1998.

13. WHO Collaborating Centre for Drug Statistics Methodology. ATC classification index with DDDs. 2020;0slo, Norw.

14. Geerlings SE, Hoepelman AIM. Immune dysfunction in patients with diabetes mellitus (DM). FEMS Immunol Med Microbiol. 1999;26(3-4):259-265.

15. Van der Linden M, Westert G, De Bakker D, Schellevis F. Tweede Nationale Studie Naar Ziekten En Verrichtingen in de Huisartsenpraktijk. Klachten En Aandoeningen in de Bevolking En in de Huisartspraktijk. Utrecht/Bilthoven: NIVEL/RIVM; 2004.

16. Smith L, Ewings P, Smith C, Thompson M, Harnden A, Mant D. Ear discharge in children presenting with acute otitis media: Observational study from UK general practice. Br J Gen Pract. 2010;60(571):101-105.

17. Venekamp RP, Prasad V, Hay AD. Are topical antibiotics an alternative to oral antibiotics for children with acute otitis media and ear discharge? BMJ. 2016;352(February):2-4.

18. Rooijackers-Lemmes E, van Balen F, Opstelten W, Wiersma T. NHG-Standaard Otitis externa (Eerste herziening). Nederlands Huisartsen Genootschap. https://www.nhg.org/standaarden/volledig/nhg-standaard-otitis-externa. Published 2014. Accessed January 15, 2020.

19. Johansson SGO, Bieber T, Dahl R, et al. Revised nomenclature for allergy for global 
use: Report of the Nomenclature Review Committee of the World Allergy Organization, October 2003. J Allergy Clin Immunol. 2004;113(5):832-836.

20. Dekker ARJ, Verheij TJM, van der Velden AW. Antibiotic management of children with infectious diseases in Dutch Primary Care. Fam Pract. 2017;34(2):169-174.

21. Dekker ARJ, Verheij TJM, van der Velden AW. Inappropriate antibiotic prescription for respiratory tract indications: Most prominent in adult patients. Fam Pract. 2015;32(4):401-407.

22. Deniz Y, Van Uum RT, De Hoog MLA, Schilder AGM, Damoiseaux RAMJ, Venekamp RP. Impact of acute otitis media clinical practice guidelines on antibiotic and analgesic prescriptions: A systematic review. Arch Dis Child. 2018;103(6):597602. 
Table 1 Baseline characteristics of included AOM episodes from 2015 to 2018

\begin{tabular}{|c|c|c|c|c|c|}
\hline & $2015-2018$ & 2015 & 2016 & 2017 & 2018 \\
\hline Total, $\mathbf{n}$ & 6667 & 1689 & 1665 & 1695 & 1618 \\
\hline Age, mean (SD) & $38.3(16.6)$ & $38.0(16.6)$ & $38.2(16.3)$ & $38.5(16.7)$ & 38.7 (16.7) \\
\hline Age, median (IQR) & $36(23)$ & $35(23)$ & $36(23)$ & $36(24)$ & $37(22)$ \\
\hline \multicolumn{6}{|l|}{ Age, n (\%) } \\
\hline $15-39$ & 3918 (58.8) & $1008(59.7)$ & $1011(60.7)$ & $977(57.6)$ & $922(57.0)$ \\
\hline $40-64$ & $2166(32.5)$ & $539(31.9)$ & $520(31.2)$ & $558(32.9)$ & 549 (33.9) \\
\hline$\geq 65$ & $583(8.7)$ & $142(8.4)$ & $134(8.0)$ & $160(9.4)$ & $147(9.1)$ \\
\hline \multicolumn{6}{|l|}{ Gender, n (\%) } \\
\hline Male & $2748(41.2)$ & 703 (41.6) & $665(39.9)$ & $706(41.7)$ & $674(41.7)$ \\
\hline Female & 3919 (58.8) & $986(58.4)$ & $1000(60.1)$ & $989(58.3)$ & $944(58.3)$ \\
\hline \multicolumn{6}{|l|}{ Comorbidities, n (\%) } \\
\hline Atopic diathesis & $1983(29.7)$ & $459(27.2)$ & $502(30.2)$ & $523(30.9)$ & 499 (30.8) \\
\hline Diabetes & $313(4.7)$ & $73(4.3)$ & $73(4.4)$ & $93(5.5)$ & $74(4.6)$ \\
\hline Use of immunosuppressive drugs ${ }^{\mathrm{a}}$, $\mathrm{n}(\%)$ & $52(0.8)$ & $16(0.9)$ & $9(0.5)$ & $17(1.0)$ & $10(0,6)$ \\
\hline
\end{tabular}

Abbreviations: AOM, acute otitis media; SD, standard deviation; IQR, interquartile range. a Defined as use of systemic corticosteroids or immunosuppressants at the day prior to AOM episode. 
Table 2 Incidence, oral antibiotic prescription and consultation rates for AOM in adults from 2015 to 2018

\begin{tabular}{|c|c|c|c|}
\hline & Incidence ratea $(95 \% \mathrm{CI})$ & Oral antibiotic prescription rate ${ }^{b}(95 \%$ CI $)$ & Consultation ratec $(95 \% \mathrm{CI})$ \\
\hline \multicolumn{4}{|c|}{ Age, years } \\
\hline $15-39$ & $7.1(6.8$ to 7.3$)$ & 44.1 (42.6 to 45.7$)$ & $1.5(1.4$ to 1.5$)$ \\
\hline $40-64$ & $4.4(4.2$ to 4.6$)$ & $49.4(47.3$ to 51.5$)$ & $1.6(1.5$ to 1.6$)$ \\
\hline$\geq 65$ & 2.7 (2.5 to 2.9$)$ & 46.1 (42.1 to 50.2$)$ & $1.7(1.6$ to 1.8$)$ \\
\hline \multicolumn{4}{|l|}{ Gender } \\
\hline Male & 4.5 (4.4 to 4.7$)$ & 43.5 (41.7 to 45.4$)$ & 1.5 (1.4 to 1.5$)$ \\
\hline Female & $6.0(5.8$ to 6.2$)$ & 47.8 (46.2 to 49.3$)$ & $1.5(1.5$ to 1.6$)$ \\
\hline \multicolumn{4}{|c|}{ Atopic diathesis } \\
\hline Yes & $7.3(7.0$ to 7.6$)$ & 44.7 (42.5 to 46.9$)$ & 1.5 (1.5 to 1.6$)$ \\
\hline No & 4.7 (4.6 to 4.9$)$ & 46.6 (45.2 to 48.0$)$ & $1.5(1.5$ to 1.6$)$ \\
\hline \multicolumn{4}{|l|}{ Diabetes } \\
\hline Yes & 4.1 (3.7 to 4.6$)$ & 46.6 (41.2 to 52.2$)$ & 1.5 (1.4 to 1.7$)$ \\
\hline No & $5.4(5.2$ to 5.5$)$ & $46.0(44.8$ to 47.2$)$ & $1.5(1.5$ to 1.6$)$ \\
\hline \multicolumn{4}{|c|}{ Use of immunosuppressive drugs ${ }^{d}$} \\
\hline Yes & $5.5(4.1$ to 7.3$)$ & $57.7(44.0$ to 70.5$)$ & 1.5 (1.2 to 1.9$)$ \\
\hline No & $5.3(5.2$ to 5.4$)$ & 45.9 (44.7 to 47.1$)$ & $1.5(1.5$ to 1.6$)$ \\
\hline
\end{tabular}

Abbreviations: AOM, acute otitis media; CI, confidence interval. a Incidence rate per 1,000 person-years. ${ }^{b}$ Prescription rates per 100 AOM episodes. ${ }^{c}$ Consultation rates per AOM episode. ${ }^{d}$ Defined as use of systemic corticosteroids or immunosuppressants at the day prior to AOM episode. 
Table 3 Consultations and additional prescriptions for AOM based on immediate antibiotic prescription (2015 - 2018)

\begin{tabular}{lcc}
\hline & Consultation rate $(95 \% \mathrm{CI})$ & Oral antibiotics during reconsultation, \% \\
\hline Oral antibiotics at first consultation & & \\
Yes & $1.6(1.6$ to 1.6$)$ & 12.0 \\
No & $1.5(1.4$ to 1.5$)$ & \\
Topical antibiotics at first consultation & & 9.3 \\
Yes & $1.6(1.5$ to 1.7$)$ & - \\
No & $1.5(1.5$ to 1.5$)$ & \\
\hline
\end{tabular}

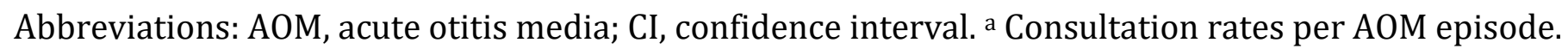




\section{Figure captions}

Figure 1: Annual incidence and prescription rates for AOM episodes from 2015 to 2018

Figure 2: Types of oral antibiotics prescribed for AOM episodes from 2015 to 2018 


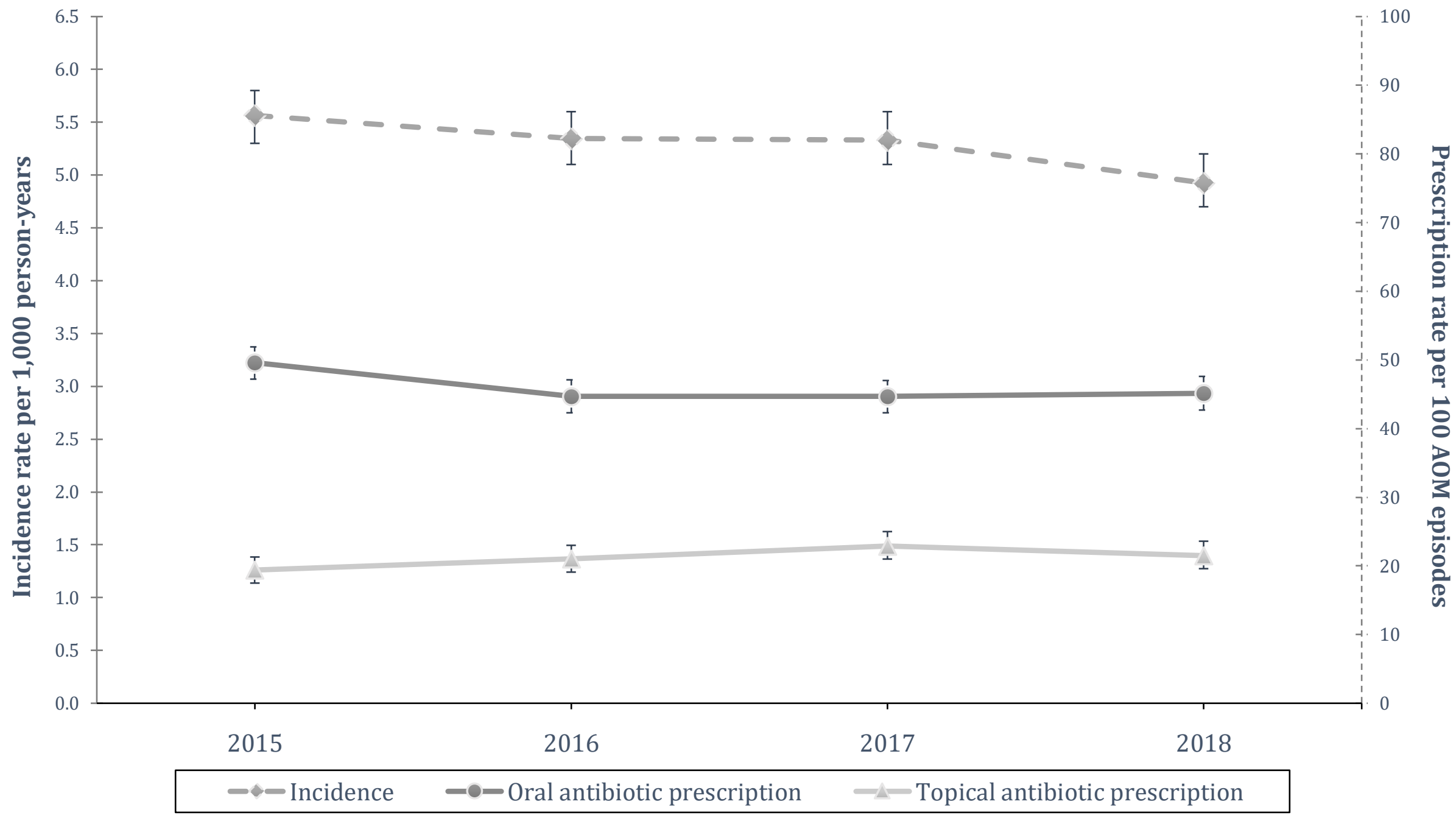




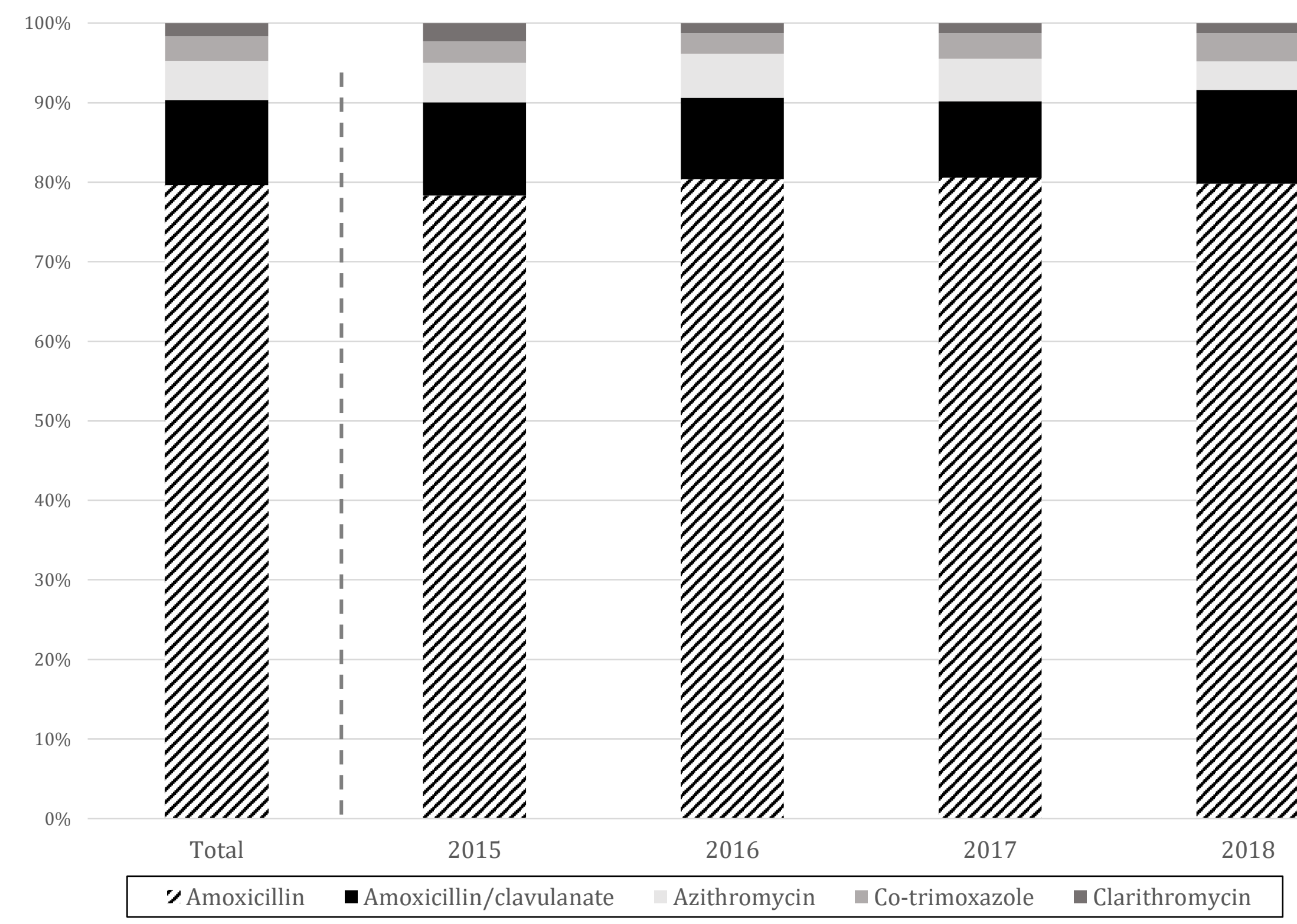

